\title{
A cosmic ray current-driven instability in partially ionised media
}

\author{
B. Reville ${ }^{1,2}$, J. G. Kirk ${ }^{1}$, P. Duffy ${ }^{2}$, and S. O'Sullivan ${ }^{2}$
}

\author{
1 Max-Planck-Institut für Kernphysik, Heidelberg 69029, Germany \\ e-mail: brian.reville@mpi-hd.mpg .de \\ 2 UCD School of Mathematical Science, Belfield, Dublin 4, Germany
}

Received 23 July 2007 / Accepted 18 September 2007

\section{ABSTRACT}

\begin{abstract}
Context. We investigate the growth of hydromagnetic waves driven by streaming cosmic rays in the precursor environment of a supernova remnant shock.

Aims. It is known that transverse waves propagating parallel to the mean magnetic field are unstable to anisotropies in the cosmic ray distribution, and may provide a mechanism to substantially amplify the ambient magnetic field. We quantify the extent to which temperature and ionisation fractions modify this picture.

Methods. Using a kinetic description of the plasma we derive the dispersion relation for a collisionless thermal plasma with a streaming cosmic ray current. Fluid equations are then used to discuss the effects of neutral-ion collisions.

Results. We calculate the extent to which the environment into which the cosmic rays propagate influences the growth of the magnetic field, and determines the range of possible growth rates.

Conclusions. If the cosmic ray acceleration is efficient, we find that very large neutral fractions are required to stabilise the growth of the non-resonant mode. For typical supernova parameters in our Galaxy, thermal effects do not significantly alter the growth rates. For weakly driven modes, ion-neutral damping can dominate over the instability at more modest ionisation fractions. In the case of a supernova shock interacting with a molecular clouds, such as in RX J1713.7-3946, with high density and low ionisation, the modes can be rapidly damped.
\end{abstract}

Key words. plasmas - instabilities - ISM: cosmic rays - ISM: supernova remnants - magnetic fields

\section{Introduction}

There is clear evidence from radio, X-ray and gamma ray observations that electrons, and probably protons, are accelerated in supernova remnants (SNR). First order Fermi acceleration is believed to be the most likely mechanism for this acceleration, as it provides a natural explanation for the spectral shape (for a recent review see Hillas 2005). Cosmic rays up to the ankle are believed to have their origin in our galaxy. However, the standard picture of Fermi acceleration must be stretched to its limit in order to accelerate protons to even the knee of the spectrum at $\sim 3 \times 10^{15} \mathrm{eV}$ (Lagage \& Cesarsky 1983). Various methods have been suggested to solve this problem in the quasilinear framework, but amplification of the ambient magnetic field probably provides the best possibility (e.g. Kirk \& Dendy 2001). The presence of bright X-ray rims near the shocks of many young supernova remnants has been interpreted as observational evidence of field amplification (Vink \& Laming 2003; Völk et al. 2005; Ballet 2006), but more detailed comparisons of these data with radio observations are necessary to confirm this result (Katz \& Waxman 2007).

Recently an attempt to derive a non-linear theory of magnetic field amplification was made by Bell \& Lucek (2001), and supported by hybrid numerical simulations (Lucek \& Bell 2000). It was shown that the cosmic ray pressure could wind up the frozen-in magnetic field to values in excess of the typically assumed self-quenching level of $\delta B / B_{0} \lesssim 1$. Building on these papers, Bell (2004) reanalysed the work of Achterberg (1983), and found a strong non-resonant mode that had been previously overlooked. Numerical MHD simulations in Bell (2004, 2005) verified that the streaming of cosmic rays can amplify the magnetic field at short lengthscales to values greater than the ambient field. The instability is driven at short wavelengths due to the thermal plasma attempting to neutralise the effects of the streaming cosmic rays. This induces a current in the thermal plasma. The cosmic rays essentially behave as unmagnetised particles and do not move coherently with magnetised thermal background particles. The same instability has also been seen, using particle in cell simulations (Niemiec \& Pohl 2007). These authors observe the non resonant instability with a considerably slower growth rate, but this may be due to the artificially low proton to electron mass ratio $\left(m_{\mathrm{p}} / m_{\mathrm{e}}=10\right)$ used in their simulations. This considerably reduces the range over which modes can be unstable. More realistic mass ratios are required to understand the true nonlinear development of this instability.

The upstream medium through which the shock-accelerated particles stream, will, in general, be incompletely ionised. Limits on the acceleration of cosmic rays in these environments have been calculated by Drury et al. (1996), including the damping of resonantly excited Alfvén waves due to friction with the neutral particles. This will also play a significant role in molecular clouds where the ionisation fractions are even lower and densities much higher. The propagation of Alfvén waves in molecular clouds has been studied by Tagger et al. (1995). Extending the work of Kulsrud \& Pearce (1969) and Zweibel \& Shull (1982), they demonstrated that the ponderomotive force of the waves can have a significant effect on the structure and resulting magnetic field within such clouds.

Recently, Bykov \& Toptygin (2005), have investigated a similar instability to that in Bell (2004), driven by a neutralising 
return current in the thermal background plasma, in a partially ionised medium. A single fluid MHD description of the background particles with a generalised Ohm's law is used, and the cosmic ray current is found by solving the kinetic transport equation. They conclude that a non-resonant mode with a rapid growth rate remains. However, as we show in Sect. 3, for high frequency waves the friction between the charged species and the neutrals is not sufficient to couple their motions, and a single fluid description of the plasma is no longer appropriate.

In the present work, we investigate various conditions that may reduce the growth of the non-resonant instability. In Sect. 2 we follow the analysis of Achterberg (1983), neglecting collisions, and quantify the extent to which thermal effects can reduce the growth of the instability. In Sect. 3 we introduce a two fluid description of the plasma which includes the collisions between the different species. The neutrals are coupled to the ions through the ion-neutral friction, and the cosmic rays are coupled to the MHD fluid through Maxwell's equations. Section 4 applies our results to SN1006 and RX J1713.7-3946. We conclude with a discussion about some of the consequences of the instability in various conditions.

\section{The kinetic non-resonant current driven instability}

The linear dispersion relation for circularly polarised transverse waves propagating parallel to the zeroth order magnetic field is

$\frac{c^{2} k^{2}}{\omega^{2}}-1=\sum_{s} \chi_{s}(k, \omega)$

where the summation is over each species with corresponding charge $q_{s}$, cyclotron frequency $\omega_{\mathrm{c} s}=q_{s} B_{0} / m_{s} c$ and plasma frequency $\omega_{\mathrm{p} s}^{2}=4 \pi e^{2} n_{s} / m_{s}$. The susceptibility $\chi_{s}$ for each component of a weakly ionised plasma is determined by integrating the Boltzmann transport equation along the unperturbed trajectories about the zeroth order field (e.g. Krall \& Trivelpiece 1973).

Previous work on the effect of streaming cosmic rays using a kinetic approach has assumed the background plasma to be collisionless (Achterberg 1983; Reville et al. 2006; Blasi \& Amato 2007). For a three-species plasma with proton electron background and proton cosmic ray component, Achterberg determined the background susceptibility for low frequency waves, $\tilde{\omega}_{s} \ll \omega_{\text {ci }}<\left|\omega_{\text {ce }}\right|$, to be

$\omega^{2} \chi_{\mathrm{bg}}=\frac{c^{2}}{\mathrm{v}_{\mathrm{A}}^{2}}\left[\tilde{\omega}_{\mathrm{i}}^{2}-\epsilon \frac{k^{2} V_{\mathrm{ti}}^{2}}{\omega_{\mathrm{ci}}} \tilde{\omega}_{\mathrm{i}}-\epsilon \frac{\omega_{\mathrm{ci}} k J_{\mathrm{cr}}^{1}}{n_{\mathrm{i}}}\right]$,

where $\tilde{\omega}_{s}=\omega-k u_{s}$ is the Doppler shifted frequency with $u_{s}$ the group drift speed of each species, $v_{\mathrm{A}}=B_{0} / \sqrt{4 \pi n_{\mathrm{i}} m_{\mathrm{i}}}$ is the Alfvén velocity and $V_{\mathrm{ti}}^{2}=k_{B} T_{\mathrm{i}} / m_{\mathrm{i}}$ the ion thermal velocity. For ease of notation we have also introduced the parameter $\epsilon$ to describe the polarisation with $\epsilon=+1$ for right-handed waves and $\epsilon=-1$ for left-handed waves $(\omega>0)$. The requirements of zero net current and quasineutrality in the shock precursor, where the proton cosmic ray component is anisotropic, induces a compensating drift in the background plasma $J_{\mathrm{cr}}^{1}=n_{\mathrm{cr}}\left(u_{\mathrm{cr}}-\omega / k\right)$, where $n_{\mathrm{cr}}$ and $u_{\mathrm{cr}}$ are the cosmic ray number density and bulk speed, respectively. We note that $n_{\text {cr }}$ is a function of distance from the shock front, since only high energy cosmic rays are expected to penetrate far into the precursor.

Using the kinetic treatment of Bell for the cosmic ray current, the resulting dispersion relation takes the form

$\tilde{\omega}_{\mathrm{i}}^{2}+\epsilon\left(\frac{k^{2} V_{\mathrm{ti}}^{2}}{\omega_{\mathrm{ci}}}\right) \tilde{\omega}_{\mathrm{i}}-v_{\mathrm{A}}^{2} k^{2}-\epsilon \zeta v_{s}^{2} \frac{k}{r_{\mathrm{gm}}}\left(\sigma\left(k r_{\mathrm{gm}}\right)-1\right)=0$, where, as appropriate in the rest frame of the upstream plasma in the precursor of a shock front, we have identified $u_{\mathrm{cr}}$ with the incoming plasma speed $v_{s}$. We define $r_{\mathrm{gm}}=p_{\min } c / e B_{0}$ the gyroradius of the minimum energy cosmic ray and consider only waves with $k>0$. The function $\sigma$ represents the cosmic ray susceptibility normalised to the return current $J_{\mathrm{cr}}^{1}$ as described in Eq. (12) of Bell (2004). We extend this result for arbitrary power law distributions $f \propto p^{-4+q}$, between $p_{\min }$ and $p_{\max }$. For $q<1$

$$
\begin{aligned}
& \sigma(x)=\frac{3}{4}\left(\frac{x^{-3}}{q-4}-\frac{x^{-1}}{q-2}\right) \ln \left|\frac{x+1}{x-1}\right|-\frac{3 x^{-2}}{2(q-4)} \\
& +\frac{3}{2(q-1)}\left(\frac{1}{q-4}-\frac{1}{q-2}\right)\left[\operatorname{Re}\left\{{ }_{2} F_{1}\left(\frac{1-q}{2}, 1, \frac{3-q}{2} ; x^{-2}\right)\right\}-1\right] \\
& +\frac{3 \pi}{4} \mathrm{i} \epsilon\left\{\begin{array}{l}
\frac{x^{-3}}{q-4}-\frac{x^{-1}}{q-2} x>1 \\
\frac{x^{1-q}}{q-4}-\frac{x^{1-q}}{q-2} x \leq 1
\end{array}\right.
\end{aligned}
$$

where ${ }_{2} F_{1}(a, b, c ; z)$ is the Gauss hypergeometric function.

In (3) we have also introduced a dimensionless parameter characterising the strength of the driving term

$\zeta=\frac{n_{\mathrm{cr}} p_{\min }}{n_{\mathrm{i}} m_{\mathrm{i}} \mathrm{v}_{\mathrm{s}}}$

In a shock precursor, we expect $p_{\min }$ to increase with distance ahead of the shock front (e.g., Eichler 1979; Blasi 2002), since higher energy particles have a larger mean free path. For $q=0$, and neglecting a logarithmic term, this leads to $n_{\mathrm{cr}} \propto 1 / p_{\text {min }}$. Thus, $\zeta$ is approximately constant, reflecting the fact that the energy density of the cosmic rays is the same in each decade of momentum. It is roughly given by $v_{s} / c$ times the ratio of the cosmic ray energy density at the shock to the incoming flux of momentum $n_{\mathrm{i}} m_{\mathrm{i}} v_{s}^{2}$. If SNR shocks are efficient in accelerating cosmic rays, one expects this ratio to be at least several per cent. (e.g., Völk et al. 1984). Hence, $\zeta \sim 0.01 v_{s} / c$. For a more detailed discussion of this quantity see Sect. 7 of Bell (2004).

The dispersion relation (3) clearly permits waves travelling in directions parallel and antiparallel to the mean magnetic field for both left and right polarised waves. For the case of parallel streaming of the cosmic rays along the magnetic field, the waves that propagate parallel the field $(\omega>0)$ are unstable, and waves propagating antiparallel $(\omega<0)$ are damped. This can be understood physically, as the Lorentz force either acting centripetally or centrifugally on the field line, depending on the polarisation of the wave. We see from (4) that $\sigma\left(k r_{\mathrm{gm}}\right)$ is a monotonically decreasing function of wavenumber for $k r_{\mathrm{gm}}>1$. The non-resonant mode emerges when $\sigma$ is negligible, and the driving term dominates over the "Alfvén term" that contains $v_{\mathrm{A}}$, i.e., when $1 \ll k r_{\text {gm }} \ll \zeta v_{s}^{2} / v_{\mathrm{A}}^{2}$.

In the frame co-moving with the drifting thermal protons, the maximum growth rate of the non-resonant mode is

$\operatorname{Im}(\omega)=\frac{\zeta}{2} \frac{v_{s}}{v_{\mathrm{A}}} \frac{v_{s}}{r_{\mathrm{gm}}} \approx \frac{1}{2} \frac{v_{s}}{v_{\mathrm{A}}} \frac{n_{\mathrm{cr}}}{n_{\mathrm{i}}} \omega_{\mathrm{ci}}$

which is independent of magnetic field strength.

For a fixed $\zeta$, the growth rate scales with the density as $n_{\mathrm{i}}^{1 / 2}$, but the mode grows on shorter length scales as $n_{\mathrm{i}}$ increases, rendering it liable to thermal damping. Defining the dimensionless temperature $\Theta=k_{\mathrm{B}} T / m_{\mathrm{i}} c^{2}$, for large $k$, and again neglecting $\sigma$, it follows from (3), in the limit $\Theta \gg v_{\mathrm{A}}^{2} / c^{2}$, that the maximum growth rate is

$\operatorname{Im}(\omega) \sim\left(n_{\mathrm{cr}} / n_{\mathrm{i}}\right)^{2 / 3}\left(\beta_{s}^{2} / \Theta\right)^{1 / 3} \omega_{\mathrm{ci}}$, 


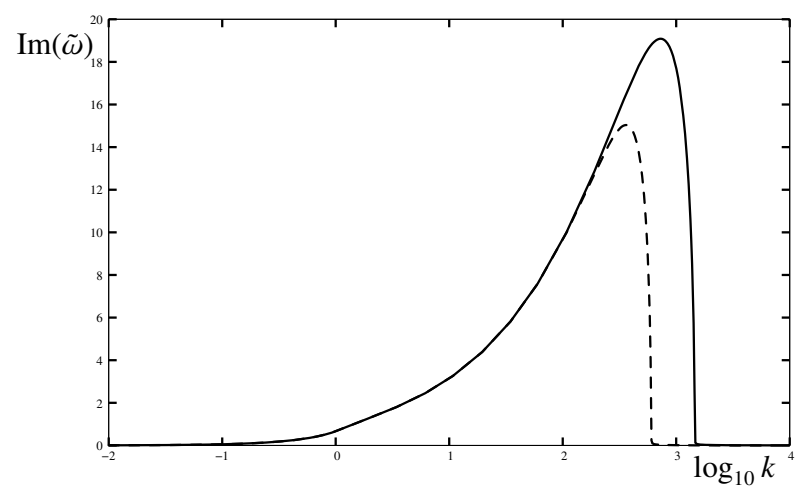

Fig. 1. $\operatorname{Im}(\tilde{\omega})$ for $\epsilon=+1$, plotted for different temperatures. $T=10^{4} \mathrm{~K}$ (solid), $T=10^{6} \mathrm{~K}$ (dashed). $k$ is plotted in units of $r_{\mathrm{gm}}^{-1}$ and $\tilde{\omega}_{\mathrm{i}}$ in units of $v_{s}^{2} / r_{\mathrm{gm}} c$. In this plot $r_{\mathrm{gm}}=10^{13} \mathrm{~cm}$ and $\zeta=0.017 v_{s} / c$ with $v_{s}=$ $5000 \mathrm{~km} \mathrm{~s}^{-1}$.

where $\beta_{s}=v_{s} / c$. This is the non-relativistic equivalent of the result found by Reville et al. (2006). At high temperatures the thermal particles behave as though they were unmagnetised. For parameters appropriate for SNR, this effect is shown in Fig.1. In order for the non-resonant mode to leave the regime of linear growth before being overtaken by the shock front, i.e., before being advected over a distance of roughly $c r_{\mathrm{gm}} / v_{s}$, one requires $\operatorname{Im}(\omega)>v_{s}^{2} / r_{\mathrm{gm}} c$. The necessary condition for thermal effects to reduce the growth rate below this value is

$\Theta>\zeta^{2} \frac{p_{\min } c}{m_{\mathrm{i}} v_{s}^{2}}$.

For typical SNR parameters this condition will only be satisfied for a very weak driving term, $\zeta \ll 1$. From Fig. 1 it is clear that damping effects do not suppress the instability in the interstellar medium of our galaxy for typical SNR shock speeds of around $5000 \mathrm{~km} \mathrm{~s}^{-1}$. Thermal effects are likely to play a more significant role for relativistic shocks, and may even provide a saturation mechanism for the current driven instability (Reville et al. 2006)

\section{The collisional non-resonant instability and ion-neutral friction effects}

Unlike the analysis of the previous section, the ideal MHD equations implicitly include the effects of collisions, although they do not appear in the final equations. Thus the analysis of Bell (2004) included the most important effects of Coulomb collisions. However, the media into which supernova shocks propagate are generally not completely ionised, and may even include weakly ionised molecular clouds. Consequently, we now extend the ideal MHD equations to include collisions between charged and neutral particles, following the approach of Tagger et al. (1995), who, in turn, extended the work of Kulsrud \& Pearce (1969). Whereas previous work involving ion-neutral friction has concentrated on shear-Alfvén waves, we consider the non-resonant modes driven by the streaming cosmic rays. These propagate along the mean field and are, therefore, circularly polarised. We continue to use a kinetic description of the cosmic rays, which remain collisionless. Neglecting viscosity and Ohmic friction, the equations of motion for a frictionally coupled system of an MHD fluid and neutrals are given by

$\frac{\mathrm{d} \boldsymbol{u}_{\mathrm{i}}}{\mathrm{d} t}=\frac{\boldsymbol{j} \times \boldsymbol{B}}{\rho_{\mathrm{i}} c}-v_{\mathrm{in}}\left(\boldsymbol{u}_{\mathrm{i}}-\boldsymbol{u}_{\mathrm{n}}\right)$,

and

$\frac{\mathrm{d} \boldsymbol{u}_{\mathrm{n}}}{\mathrm{d} t}=-v_{\mathrm{ni}}\left(\boldsymbol{u}_{\mathrm{n}}-\boldsymbol{u}_{\mathrm{i}}\right)$,
(Tagger et al. 1995), where $\boldsymbol{j}$ is the current carried by the plasma (excluding the cosmic rays), $\boldsymbol{u}_{\mathrm{i}}$ and $\boldsymbol{u}_{\mathrm{n}}$ are the velocities of the ionised and neutral components, respectively, and $v_{\text {in }}$ and $v_{\mathrm{ni}}$ are the momentum exchange frequencies. In writing these equations we have neglected all terms of order $m_{\mathrm{e}} / m_{\mathrm{i}, \mathrm{n}}$. The cosmic ray current is contained in Ampere's law $\nabla \times \boldsymbol{B}=(4 \pi / c)\left(\boldsymbol{j}+\boldsymbol{j}_{\mathrm{cr}}\right)$. The momentum exchange frequencies are related by $\rho_{\mathrm{n}} \nu_{\mathrm{ni}}=\rho_{\mathrm{i}} \nu_{\mathrm{in}}$, where $\rho_{\mathrm{n}}$ and $\rho_{\mathrm{i}}$ are the densities of the neutral and ionised components. Kulsrud \& Cesarsky (1971) give the following approximation for plasmas in the temperature range $10^{2} \mathrm{~K}<T<10^{5} \mathrm{~K}$

$v_{\text {in }} \approx 8.9 \times 10^{-9} n_{\mathrm{n}}\left(\frac{T}{10^{4} \mathrm{~K}}\right)^{0.4} \mathrm{~s}^{-1}$.

The resulting dispersion relation is easily found to be

$\omega^{2}\left(1+\frac{i v_{\mathrm{in}}}{\omega+i v_{\mathrm{ni}}}\right)=k^{2} \mathrm{v}_{\mathrm{A}}^{2}+\epsilon \zeta \frac{\mathrm{v}_{\mathrm{s}}^{2}}{r_{\mathrm{gm}}} k(\sigma-1)$.

In the high frequency limit, $|\omega| \gg v_{\text {in }}$, this reduces to a dispersion relation similar to (3), although the term representing thermal effects is absent. In the opposite limit $|\omega| \ll v_{\text {ni }}$ we find

$\omega^{2} \approx \frac{\rho_{\mathrm{i}}}{\rho}\left[k^{2} v_{\mathrm{A}}^{2}+\epsilon \zeta \frac{v_{s}^{2}}{r_{\mathrm{gm}}} k(\sigma-1)\right]$

For high collision frequencies, the neutral and ionised components are tied together and the effect of ion-neutral collisions is simply to increase the effective mass of the ions.

For strongly driven, non-resonant modes (12) reduces to

$\omega\left(\omega^{2}+\zeta \frac{v_{s}^{2}}{r_{\mathrm{gm}}} k\right)+\mathrm{i} v_{\mathrm{in}}\left[(1+Z) \omega^{2}+Z \zeta \frac{v_{s}^{2}}{r_{\mathrm{gm}}} k\right]=0$,

where $Z=\rho_{\mathrm{i}} / \rho_{\mathrm{n}}=v_{\mathrm{ni}} / \nu_{\text {in }}$. For all physically relevant parameters (i.e., $\zeta, Z>0$ ), this cubic polynomial in $\omega$ has three purely imaginary roots, two of which are damped modes, and the third the non-resonant growing mode. Thus, ion-neutral collisions are unable to stabilise the strongly driven mode, although they can affect its growth rate.

The general analytic expressions for the growing mode are cumbersome, but various limiting cases yield interesting results. In the limit of low ionisation, $Z \gg 1$ one recovers the growth rate given in (6). For $Z \ll 1$ the growth rate is given by

$\gamma=-\frac{v_{\mathrm{in}}}{2}+\frac{1}{2} \sqrt{v_{\mathrm{in}}^{2}+4 \zeta \frac{v_{s}^{2}}{r_{\mathrm{gm}}} k}$

If $v_{\text {in }}^{2} \ll 4 \zeta v_{s}^{2} k / r_{\text {gm }}$ collisions are too slow to compete with the driving, and the growth rate is again the same as (6) with only a small reduction due to collisions. However, in addition to the low frequency limit in which the components are tied together (13), a new regime arises. If the collisional drag on the ions is sufficient to affect the driving, but not to couple the components, i.e., $v_{\text {in }}^{2} \gg 4 \zeta v_{s}^{2} k / r_{\mathrm{gm}} \gg v_{\mathrm{ni}}^{2}$, then we obtain a similar expression to that of Bykov \& Toptygin (2005),

$\gamma \approx \zeta \frac{v_{s}^{2}}{v_{\mathrm{in}} r_{\mathrm{gm}}} k=\frac{\omega_{\mathrm{ci}}}{v_{\mathrm{in}}} \frac{n_{\mathrm{cr}}}{n_{\mathrm{i}}} k v_{s}$

However, this applies only when $\gamma \ll v_{\text {in }}$.

In Fig. 2 we illustrate the influence of ion-neutral collisions for SNR parameters. Thermal damping is negligible, but a weak dependence on temperature enters via Eq. (11). Using (12), we plot the maximum growth rate, as a function of ionisation fraction $x_{\mathrm{i}} \equiv n_{\mathrm{i}} / n$, taking the driving term $\zeta=0.01 x_{\mathrm{i}} v_{s} / c$ since the 


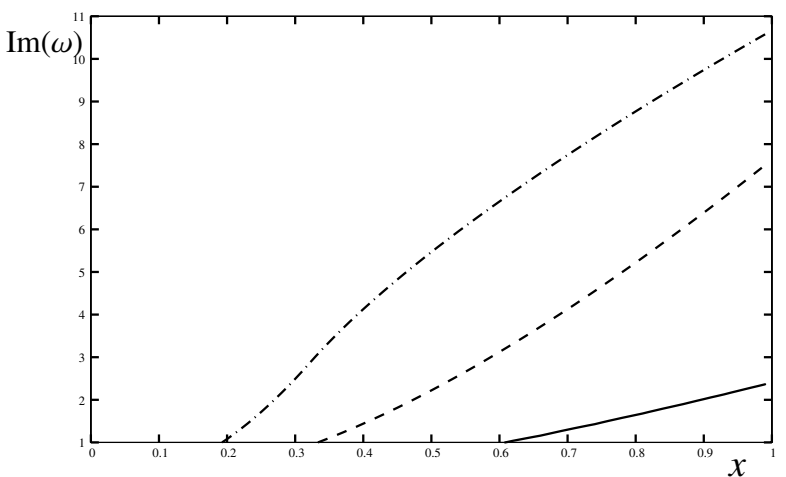

Fig. 2. Maximum growth rate as a function of ionisation fraction for total density $n=n_{\mathrm{i}}+n_{\mathrm{n}}$ in a H-H $\mathrm{H}^{+}$gas: $0.1 \mathrm{~cm}^{-3}, 10^{4} \mathrm{~K}$ (solid), $1.0 \mathrm{~cm}^{-3}$, $10^{3} \mathrm{~K}$ (dash), $10.0 \mathrm{~cm}^{-3}, 10^{2} \mathrm{~K}$ (dash-dot). We take $\zeta=0.01 x_{\mathrm{i}} v_{s} / c$ with shock speed $v_{s}=5000 \mathrm{~km} \mathrm{~s}^{-1}$, and $r_{\mathrm{gm}}=10^{14} \mathrm{~cm} . \omega$ is in units of $v_{s}^{2} / r_{\text {gm }} c$. Once the $\omega_{\max } \leq 1$ the non-resonant instability is not effective in amplifying the magnetic field.

cosmic rays couple directly only to the ionised component. For higher density plasmas, a larger fraction of neutral particles is necessary to reduce the growth rate below the threshold value of $v_{s}^{2} / r_{\mathrm{gm}} c$.

\section{Application to SNR}

X-ray observations of several young supernovae display bright rims immediately downstream of the outer shock. This has been modelled as the synchrotron cooling of relativistic electrons, which requires a magnetic field substantially higher than in the surrounding medium (Vink \& Laming 2003; Ballet 2006; Völk et al. 2005). In the case of SN 1006 the magnetic field at the shock front is estimated to be of the order $100 \mu \mathrm{G}$. One way in which field amplification may take place is if the non-resonant instability discussed above occurs upstream of the shock front. Since this instability depends on strong driving by cosmic rays, it cannot bootstrap from an initial state in which they are absent. Nevertheless, we can check its consistency, by requiring that, given a substantial cosmic ray pressure at the shock, the growth rate is sufficiently rapid for it to enter the nonlinear regime before being overtaken by the shock. Thus, the physical conditions far upstream $\left(B, n, x_{\mathrm{i}}, T\right)$ together with the shock speed $\mathrm{v}_{\mathrm{s}}$ and cosmic ray intensity $U_{\mathrm{cr}} / \rho_{\mathrm{i}} v_{s}^{2}=0.1$, should combine to yield a growth rate in excess of $v_{s}^{2} /\left(r_{\mathrm{gm}} c\right)$.

In the case of SN1006, the shock velocity is approximately $v_{s} \approx 2900 \mathrm{~km} \mathrm{~s}^{-1}$ the density is $0.05 \lesssim n \lesssim 0.25 \mathrm{~cm}^{-3}$, and the neutral fraction is small, $x_{\mathrm{i}} \approx 0.9$ (Raymond et al. 2007). Taking $n_{\mathrm{i}}=0.25 \mathrm{~cm}^{-3}$ gives $\zeta v_{s}^{2} / v_{\mathrm{A}}^{2} \sim 10$. The low density and shock speed, imply that the non-resonant mode is not very strongly driven, but the driving is nevertheless strong compared to the collision frequency. Very close to the shock, the growth time of the field is on the order of years, while further from the shock the growth rate decreases quite dramatically, as the number density of cosmic rays decreases. For the parameters given above the maximum growth rate is $\sim 10 v_{s}^{2} / r_{\mathrm{gm}} c$. The non-resonant mode may still amplify the field into the nonlinear regime.

The low neutral fraction in SN1006 means that ion-neutral collisions are not likely to dramatically reduce the instability. The interaction of a supernova blast wave with a molecular cloud, such as that observed close to the north-western rim of RX J1713.7-3946 would have a much more significant effect on the growth. Although the exact parameters are uncertain, molecular clouds are generally clumpy with interclump densities in the range $5-25 \mathrm{~cm}^{-3}$ and ionisation fractions not larger than $10 \%$ (Chevalier 1999). Adopting the values in Malkov et al. (2005) $T=10^{2} \mathrm{~K}, n=23 \mathrm{~cm}^{-3}, x_{\mathrm{i}}=0.01, v_{s}=10^{8} \mathrm{~cm} \mathrm{~s}^{-1}$ the collision frequency is larger than the growth rate. The nonresonant driving term is unable to dominate over the Alfvén term and growth is driven resonantly by the cosmic rays themselves, at very long wavelengths $k r_{\text {gm }} \ll 1$. At shorter length scales, the waves are rapidly ion-neutral damped Alfvén waves. The shortest growth timescale is on the order of $\sim 10^{6} \mathrm{yrs}$. This value varies with choice of $r_{\mathrm{gm}}$, temperature and density, but for high density and low ionisation fractions, the growth timescale is longer than the free expansion phase of a supernova remnant, or the lifetime of the wave in the shock precursor, suggesting that the Fermi acceleration mechanism may switch off upon interaction with a molecular cloud.

\section{Discussion}

It has been suggested that the non-resonant cosmic ray current driven instability may play a significant role in the acceleration of cosmic rays (Bell 2004). In the case of strongly driven unstable modes, the mean field is unable to quench the growth of the waves at $\delta B \lesssim B_{0}$. The maximum achievable energy may be pushed beyond the Lagage-Cesarsky limit (Lagage \& Cesarsky 1983), not only by producing nonlinear turbulence to scatter the particles but also through amplification of the ambient field.

In this paper we have shown that the non-resonant instability can have a very rapid growth rate under most young SNR conditions, in the interstellar medium. Thermal effects are of little importance for nonrelativistic shocks, and large neutral fractions are required to prevent growth beyond the linear regime. However, efficient injection and acceleration are essential for this mechanism to occur. The largest uncertainty in our models arises in determining the strength of the driving term $\zeta$, which in our models depends only on ionisation fraction and not explicitly on the ion density. As a result the instability increases in more dense regions. It should be noted however, that energy losses close to the injection momentum can reduce cosmic ray production in over dense regions (Drury et al. 1996).

The nonlinear evolution of field amplification via this instability requires further study. Current MHD simulations have only been performed with constant cosmic ray currents (Bell $2004,2005)$, using periodic boundary conditions. The first results of PIC simulations of this mechanism have recently been published (Niemiec \& Pohl 2007). The dynamical range over which the non-resonant instability operates is dependent on the mass differences between magnetised and unmagnetised particles. Realistic proton electron mass ratios are essential in simulating this process. This is most likely the reason for the relatively low saturation value of $\delta B \sim B_{0}$. As the field becomes highly nonlinear, $\delta B>B_{0}$, the cosmic ray trajectories will be deflected. Future PIC simulations will give further insight the evolution and saturation of the non-resonant instability.

Both the numerical MHD simulations of Bell (2004, 2005) and the PIC simulations of Niemiec \& Pohl (2007) show that in the initial stages of the instability's development cavities and filaments are created along the direction of the mean magnetic field. It is clear from equation (10) that the resulting cavities would be mostly populated by neutral species. This is in contrast with the results of Tagger et al. (1995), where the wave damping expels the neutrals from ionised flux tubes, although the waves they considered were not driven by any mechanism. In highly dense, low ionisation regions, where the instability cannot exceed the damping, the analysis of Tagger et al. (1995) applies. 
The connection between magnetic field amplification and the broadening of the narrow component $\mathrm{H} \alpha$ line has been discussed by Ghavamian et al. (2007). These authors suggest that an observed broad component, of width $\delta v$, such that $\delta v / v_{\mathrm{A}}>1$, implies a turbulent field component $\delta B / B>1$. We have demonstrated that the cosmic ray current driven instability is still capable of amplifying fields in the Balmer dominated shocks. This may provide a possible observational test of the amplification of the turbulent magnetic component in supernova shocks.

Acknowledgements. This research was jointly supported by COSMOGRID and the Max-Planck-Institut für Kernphysik, Heidelberg. We thank the referee for several insightful suggestions. B.R. would like to thank Prof. A. R. Bell for many helpful discussions.

\section{References}

Achterberg, A. 1983, A\&A, 119, 274

Ballet, J. 2006, Adv. Space Res., 37, 1902

Bell, A. R. 2004, MNRAS, 353, 550

Bell, A. R. 2005, MNRAS, 358, 181

Bell, A. R., \& Lucek, S. G. 2001, MNRAS, 321, 433

Blasi, P. 2002, Astropart. Phys., 16, 429
Blasi, P., \& Amato, E. 2007, [arXiv:0706. 1722]

Bykov, A. M., \& Toptygin, I. N. 2005, Astron. Lett. 31, 748

Chevalier, R. A. 1999, ApJ, 511, 798

Drury, L. O. C., Duffy, P., \& Kirk, J. G. 1996, A\&A, 309, 1002

Eichler, D. 1979, ApJ, 229, 419

Ghavamian, P., Laming, J. M., \& Rakowski, C. E. 2007, ApJ, 654, L69

Hillas, A. M. 2005, J. Phys. G Nucl. Phys., 31, 95

Katz, B., \& Waxman, E. 2007, [arXiv:0706.3485]

Kirk, J. G., \& Dendy, R. O. 2001, J. Phys. G Nucl. Phys., 27, 1589

Krall, N. A., \& Trivelpiece, A. W. 1973, Principles of plasma physics (McGrawHill)

Kulsrud, R., \& Pearce, W. P. 1969, ApJ, 156, 445

Kulsrud, R. M., \& Cesarsky, C. J. 1971, Astrophys. Lett., 8, 189

Lagage, P. O., \& Cesarsky, C. J. 1983, A\&A, 125, 249

Lucek, S. G., \& Bell, A. R. 2000, MNRAS, 314, 65

Malkov, M. A., Diamond, P. H., \& Sagdeev, R. Z. 2005, ApJ, 624, L37

Niemiec, J., \& Pohl, M. 2007, [arXiv:0705 . 4459]

Raymond, J. C., Korreck, K. E., Sedlacek, Q. C., et al. 2007, ApJ, 659, 1257

Reville, B., Kirk, J. G., \& Duffy, P. 2006, Plasma Physics and Controlled Fusion, 48, 1741

Tagger, M., Falgarone, E., \& Shukurov, A. 1995, A\&A, 299, 940

Vink, J., \& Laming, J. M. 2003, ApJ, 584, 758

Völk, H. J., Drury, L. O., \& McKenzie, J. F. 1984 A\&A, 130, 19

Völk, H. J., Berezhko, E. G., \& Ksenofontov, L. T. 2005, A\&A, 433, 229

Zweibel, E. G., \& Shull, J. M. 1982, ApJ, 259, 859 